\title{
PROJECTIVE LIMIT OF INFINITE RADON MEASURES
}

\author{
SUSUMU OKADA AND YOSHIAKI OKAZAKI \\ (Received 13 April; revised 3 June 1977) \\ Communicated by E. Strzelecki
}

\begin{abstract}
We show that for any self-consistent sequentially maximal system $\left\{\mu_{\alpha}\right\}$ of infinite (perhaps non- $\sigma$-finite) Radon measures, the projective limit of $\left\{\mu_{\alpha}\right\}$ exists.
\end{abstract}

Subject classification (Amer. Math. Soc. (MOS) 1970): primary 28A35.

\section{Introduction}

Yamasaki (1975) has studied the extension problem of a self-consistent system of infinite measures to a $\sigma$-additive measure, under the assumption that every system of probability measures has a unique $\sigma$-additive extension. In this paper we consider a system of infinite Radon measures on arbitrary topological spaces. In Theorem 1, we prove that for every denumerable projective system $\left\{\mu_{n}\right\}$, there uniquely exists a Radon measure $\mu$ satisfying $p_{n}(\mu)=\mu_{n}$. In Theorem 2, for a general projective system, we show that Kolmogorov consistency theorem is valid.

Throughout this paper we assume every topological space is a Hausdorff space. Let $T$ be a topological space. By the Borel field $\mathbf{B}(T)$, we mean the minimal $\sigma$-algebra generated by all open subsets of $X$. A Radon measure $\mu$ is a non-negative, extended real-valued Borel measure on $\mathbf{B}(T)$ such that

1) for $x$ in $T$, there exists an open neighborhood $U$ of $x$ such that $\mu(U)<\infty$ (locally finite);

2) for every Borel set $E$ in $\mathbf{B}(T)$,

$$
\mu(E)=\sup \{\mu(K) ; K \subset E \text { and } K \text { is compact }\} .
$$

Let $(A, \leqq)$ be a directed set. A system $\left\{\left(T_{\alpha}, p_{\alpha \beta}\right) ; \alpha, \beta \in A, \alpha \leqq \beta\right\}$ of topological spaces $\left\{T_{\alpha} ; \alpha \in A\right\}$ is said to be a projective system if for $\alpha \leqq \beta \leqq \gamma, p_{\alpha \beta}$ and $p_{\beta \gamma}$ are continuous $\left(p_{\alpha \alpha}=\mathrm{id}\right)$ and $p_{\alpha \beta} \circ p_{\beta \gamma}=p_{\alpha \gamma}$ holds. We 
denote by $T=\lim _{\leftarrow_{\alpha}} T_{\alpha}$ the projective limit $\left\{\left(x_{\alpha}\right) \in \prod_{\alpha \in A} T_{\alpha} ; p_{\alpha \beta}\left(x_{\beta}\right)=x_{\alpha}, \alpha \leqq\right.$ $\beta\}$. By $p_{\alpha}$ we mean the projection of $T$ to $T_{\alpha}$. A family $\left\{\mu_{\alpha}\right\}$ of measures on the projective system $\left\{\left(T_{\alpha}, p_{\alpha}, p_{\alpha \beta}\right)\right\}$ is called self-consistent if $\mu_{\alpha}$ is a nonnegative, extended real-valued Borel measure on $T_{\alpha}$ and $p_{\alpha \beta}\left(\mu_{\beta}\right)=\mu_{\alpha}$ holds for $\alpha \leqq \beta$.

\section{Kolmogorov consistency theorem}

THEOREM 1. Let $T=\lim _{\leftarrow_{n}} T_{n}$ be the projective limit of a projective system $\left\{\left(T_{n}, p_{n}, p_{n m}\right) ; n \leqq m\right\}$ and $\left\{\mu_{n}\right\}$ be a self-consistent system of Radon measures on $\left\{T_{n}\right\}$. Then there exists a unique Radon measure $\mu$ on $T$ such that $p_{n}(\mu)=\mu_{n}$ on $T_{n}$.

Proof. Let $\left\{U_{\lambda} ; \lambda \in \Lambda\right\}$ be the family of all open subsets of $T_{1}$ such that $\mu_{1}\left(U_{\Lambda}\right)<\infty$. Naturally we may assume $\Lambda$ is directed. For any $\lambda$ the system $\left\{\left(p_{\text {In }}^{-1}\left(U_{\lambda}\right), q_{n m}\right) ; n \leqq m\right\}$ is a projective system, where $q_{n m}=p_{n m} \mid p_{1 m}^{-1}\left(U_{\lambda}\right)$. If we put $\nu_{\lambda}^{n}=\mu_{n} \mid p_{\ln }^{-1}\left(U_{\lambda}\right)$, then we have $q_{n m}\left(\nu_{\lambda}^{m}\right)=\nu_{\lambda}^{n}$ for $n \leqq m$, where $\mu_{n} \mid p_{\ln }^{-1}\left(U_{\lambda}\right)$ is the restriction of $\mu_{n}$ to $p_{\ln }^{-1}\left(U_{\lambda}\right)$. By Theorem 4.2 in Bourbaki (1969), there exists a Radon measure $\nu_{\lambda}$ on $V_{\lambda}=\lim _{\leftarrow_{n}} p_{\ln }^{-1}\left(U_{\lambda}\right)$ for every $\lambda$ in $\Lambda$. From the construction of $V_{\lambda}$, it is clear $V_{\lambda}=p_{1}^{-1}\left(U_{\lambda}\right)$. We put

$$
\mu(E)=\sup _{\lambda} \nu_{\lambda}\left(E \cap V_{\lambda}\right)
$$

for every Borel set $E$ in $\mathbf{B}(T)$. For every Borel set $E_{n}$ in $\mathbf{B}\left(T_{n}\right)$, we have

$$
\begin{aligned}
p_{n}(\mu)\left(E_{n}\right) & =\mu\left(p_{n}^{-1}\left(E_{n}\right)\right)=\sup _{\lambda} \nu_{\lambda}\left(p_{n}^{-1}\left(E_{n}\right) \cap V_{\lambda}\right) \\
& =\sup _{\lambda} \nu_{\lambda}\left(p_{n}^{-1}\left(E_{n} \cap p_{1 n}^{-1}\left(U_{\lambda}\right)\right)\right) \\
& =\sup _{\lambda} \nu_{\lambda}^{n}\left(E_{n} \cap p_{1 n}^{-1}\left(U_{\lambda}\right)\right) \\
& =\sup _{\lambda} \mu_{n}\left(E_{n} \cap p_{1 n}^{-1}\left(U_{\lambda}\right)\right) \\
& =\sup _{\lambda}\left(\mu_{n} \mid E_{n}\right)\left(E_{n} \cap p_{1 n}^{-1}\left(U_{\lambda}\right)\right) \\
& =\mu_{n}\left(E_{n}\right),
\end{aligned}
$$

since $\mu_{n} \mid E_{n}$ is a Radon measure on $E_{n}$. Therefore we have $p_{n}(\mu)=\mu_{n}$ for every $n$, which shows $\mu$ is locally finite. Since every $\nu_{\lambda}$ is a Radon measure, $\mu$ is a Radon measure on $T$. 
Assume $\nu$ is another Radon measure on $T$ such that $p_{n}(\nu)=\mu_{n}$. For every compact subset $K$ of $T$, it holds $K=\bigcap_{n=1}^{\infty} p_{n}^{-1} p_{n}(K)$ (see Proposition 4.2 in Bourbaki (1969)). Thus we have

$$
\begin{aligned}
\nu(K) & =\lim _{n \rightarrow \infty} \nu\left(p_{n}^{-1} p_{n}(K)\right) \\
& =\lim _{n \rightarrow \infty} \mu_{n}\left(p_{n}(K)\right) \\
& =\mu(K),
\end{aligned}
$$

which shows $\nu$ is identical to $\mu$. This proves the theorem.

COROLLARY. If $\mu_{n}$ is $\sigma$-finite for some $n$, then the Radon measure $\mu$ is also $\sigma$-finite, moreover $\mu$ is outer regular.

Proof. Clearly $\mu$ is $\sigma$-finite and satisfies the conditions of Theorem A in Amemiya, Okada and Okazaki (to appear).

Next we deal with the general projective system $\left\{\left(T_{\alpha}, p_{\alpha}, p_{\alpha \beta}\right) ; \alpha \leqq \beta\right\}$. If each $\mu_{\alpha}$ is a probability measure, then $\left\{\mu_{\alpha}\right\}$ has a unique $\sigma$-additive extension by Theorem 5.1.1 in Bochner (1955). We put

$$
D(A)=\left\{\left(\alpha_{n}\right)_{n-1}^{\infty} ; \quad \alpha_{1}<\alpha_{2}<\cdots, \alpha_{n} \in A\right\} .
$$

For every $M=\left(\alpha_{n}\right)_{n=1}^{\infty}$ in $D(A)$, we denote by $p_{M}$ the natural projection of $T$ to $T_{M}=\lim _{-_{n}} T_{\alpha_{n}}$. We say the projective system $\left\{\left(T_{\alpha}, p_{\alpha}, p_{\alpha \beta}\right) ; \alpha \leqq \beta\right\}$ is sequentially maximal if $p_{M}$ is a surjection for every $M$ in $D(A)$.

THEOREM 2. Let $T=\lim _{\leftarrow_{\alpha}} T_{\alpha}$ be the projective limit of a sequentially maximal projective system $\left\{\left(T_{\alpha}, p_{\alpha}, p_{\alpha \beta}\right) ; \alpha \leqq \beta\right\}$ such that $p_{\alpha}$ is surjective. Let $\left\{\mu_{\alpha}\right\}$ be a self-consistent system of Radon measures on $\left\{T_{\alpha}\right\}$. Then there exists a unique $\sigma$-additive measure on the $\sigma$-algebra $\mathbf{B}_{1}=\bigcup_{M \in D(A)} P_{M}^{-1}\left(\mathbf{B}\left(T_{M}\right)\right)$ satisfying that $p_{M}(\mu)$ is a Radon measure on $T_{M}$ and $p_{\alpha}(\mu)=\mu_{\alpha}$ on $T_{\alpha}$ for every $\alpha$ in $A$.

Proof. Let $\mathbf{F}$ be the algebra $\bigcup_{\alpha \in A} p_{\alpha}^{-1}\left(\mathbf{B}\left(T_{\alpha}\right)\right)$. We define a finitely additive set function $\rho$ on $\mathbf{F}$ by

$$
\rho\left(p_{\alpha}^{-1}\left(E_{\alpha}\right)\right)=\mu_{\alpha}\left(E_{\alpha}\right)
$$

for every $E_{\alpha}$ in $\mathbf{B}\left(T_{\alpha}\right)$. Since $p_{\alpha}$ is surjective, $\rho$ is well defined.

For every $M=\left(\alpha_{n}\right)_{n=1}^{\infty}$ in $D(A), \quad\left\{\mu_{\alpha_{n}}\right\}$ is self-consistent on $\left\{\left(T_{\alpha_{n}}, q_{n M}, p_{\alpha_{n} \alpha_{m}}\right)\right\}$, where $q_{n M}$ is the natural projection of $T_{M}$ to $T_{\alpha_{n}}$. By Theorem 1 there exists a unique Radon measure $\mu_{M}$ on $T_{M}$ such that $q_{n M}\left(\mu_{M}\right)=\mu_{\alpha_{n}}$. We introduce an order relation in $D(A)$ as follows: for $M=\left(\alpha_{n}\right)_{n=1}^{\infty}, N=\left(\beta_{n}\right)_{n=1}^{\infty}$ in $D(A)$, 
$M \leqq N$ if and only if $\alpha_{n} \leqq \beta_{n}$ for every $n$.

For $\left(x_{\beta_{n}}\right)$ in $T_{N}$ we set $p_{M N}\left(\left(x_{\beta_{n}}\right)\right)=\left(p_{\alpha_{n} \beta_{n}}\left(x_{\beta_{n}}\right)\right)$. It follows that $p_{M}=p_{M N} p_{N}$, and $p_{M N}\left(\mu_{N}\right)=\mu_{M}$ since it holds $q_{n M} p_{M N}\left(\mu_{N}\right)=p_{\alpha_{n} \beta_{n}} q_{n N}\left(\mu_{N}\right)=p_{\alpha_{n} \beta_{n}}\left(\mu_{\beta_{n}}\right)=\mu_{\alpha_{n}}$. Thus we can define $\mu$ as follows:

$$
\mu\left(p_{M}^{-1}\left(E_{M}\right)\right)=\mu_{M}\left(E_{M}\right)
$$

for every $E_{M}$ in $\mathbf{B}\left(T_{M}\right)$. Obviously $\mu$ is $\sigma$-additive on $\mathbf{B}_{1}$ and $p_{M}(\mu)$ is equal to the Radon measure $\mu_{M}$ on $\mathbf{B}\left(T_{M}\right)$ for every $M$ in $D(A)$. For each $\alpha$ in $A$, there exists an $M$ in $D(A)$ such that $\alpha_{1}=\alpha$, which shows $p_{\alpha}(\mu)=q_{1 M} p_{M}(\mu)=$ $q_{1 M}\left(\mu_{M}\right)=\mu_{\alpha_{1}}=\mu_{\alpha}$. Since $\mathbf{B}_{1}$ is a $\sigma$-algebra containing $\mathbf{F}, \mu$ is a $\sigma$-extension of $\rho$.

Suppose $\nu$ is another $\sigma$-additive extension of $\rho$ on $\mathbf{B}_{1}$ such that $p_{M}(\nu)$ is a Radon measure on $T_{M}$ for every $M=\left(\alpha_{n}\right)_{n=1}^{\infty}$ in $D(A)$. Then it follows that for every $n, q_{n M}\left(p_{M}(\nu)\right)=p_{\alpha_{n}}(\nu)=p_{\alpha_{n}}(\rho)=p_{\alpha_{n}}(\mu)=q_{n M}\left(p_{M}(\mu)\right)$ on $T_{\alpha_{n}}$. Thus by Theorem 1, we have $p_{M}(\nu)=p_{M}(\mu)$. From the definition of $\mathbf{B}_{1}, \nu$ is equal to $\mu$. This proves the theorem.

Remark. In Theorem $2, \mu$ is not necessarily extended to a $\tau$-smooth Borel measure even if every $\mu_{\alpha}$ is a probability measure (see Theorem 4.6 in Moran (1968)).

\section{REFERENCES}

I. Amemiya, S. Okada and Y. Okazaki (to appear), 'Pre-Radon measures on topological spaces'.

S. Bochner (1955), Harmonic analysis and the theory of probability (University of California Press).

N. Bourbaki (1969), Integration Chapter 9 (Hermann, Paris).

W. Moran (1968), 'The additivity of measures on completely regular spaces', J. London Math. Soc. 43, 633-639.

Y. Yamasaki (1975), 'Kolmogorov's extension theorem for infinite measures', Publ. RIMS. Kyoto Univ. 10, 381-411.

Department of Mathematics, Institute of Advanced Studies, The Australian National University,

P.O. Box 4, Canberra, A.C.T. 2600, Australia.

Department of Mathematics,

Kyushu University 33,

Fukuoka 812, Japan. 